\title{
Modélisation hydrodynamique de la dispersion à court terme dans une mer macrotidale: validation par des mesures à haute résolution de radiotraceurs solubles
}

\author{
(a) (b) \\ Pascal Bailly du Bois , Franck Dumas \\ (a) \\ Institut de Radioprotection et de Sûreté Nucléaire, Laboratoire de Radioécologie de \\ Cherbourg-Octeville (IRSN/DEI/SECRE/LRC), pascal.bailly-du-bois@irsn.fr \\ (b) \\ IFREMER/DEL Applications Opérationnelles,fdumas@ifremer.fr
}

\section{Résumé}

Le programme DISPRO a pour objectif de valider les paramètres de dispersion de modèles hydrodynamiques par des données de terrain pour le court terme (de l'heure à la semaine) et les petites distances (de $1000 \mathrm{~m}$ à $30 \mathrm{~km}$ ). Ce programme s'appuie sur un modèle de courants instantanés 2D de maille $110 \mathrm{~m}$, associé à des mesures de terrain acquises à haute fréquence dans le panache d'un émissaire de rejet. A ce stade des travaux, les mesures confirment la représentativité du modèle durant les six heures qui suivent un rejet. Cette validation permettra de disposer de modèles ayant des incertitudes connues pour la simulation de la dispersion de substances solubles, pour des conditions réalistes de rejets chroniques ou accidentels en mers macrotidales.

\begin{abstract}
DISPRO project aims to validate hydrodynamic models dispersion parameters by field data for the short-term (hours to week) and short distances (100 meters to $30 \mathrm{~km}$ ). This project is based on a 2D instantaneous currents model with a mesh size of $110 \mathrm{~m}$, coupled with high frequency field data collected in the release plume. At this step of the works, measurements confirm the reliability of the model during six hours following a release. This validation made possible developpement of models with known incertitude to simulate dispersion of dissolved substances in realistic conditions for normal or accidental situations in macrotidal seas.
\end{abstract}

Mots-clés : modèle, Manche, dispersion, tritium, validation

\section{Introduction}

L'évaluation de l'impact de rejets de substances polluantes en milieu marin, que ce soit en situation normale ou accidentelle, apparait comme un besoin de plus en plus aigu dans les mers européennes : une part importante du commerce mondial y transite et de nombreux émissaires de rejet industriels y aboutissent. Des modèles hydrodynamiques de dispersion de substances solubles ont été développés, qui reproduisent les courants instantanés ou résiduels de marée avec des emprises et des résolutions de calcul très variables. 
Cependant les modèles d'advection et de dispersion ne disposent pas de données de terrain qui permettent de les valider avec précision dans l'environnement proche d'un émissaire de rejet pour des durées s'étendant de l'heure à la semaine et des distances de 100 mètres à $30 \mathrm{~km}$.

Les mesures de traceurs in-situ ont permis de bien caler les modèles de dispersion à moyen et long terme et d'aboutir à une représentation réaliste de la dynamique des masses d'eaux depuis la semaine jusqu'à plusieurs années ${ }^{1,2,3}$. Des données comparables peuvent être acquises pour valider un modèle de dispersion en champ proche.

L'objectif du présent travail est de préciser un jeu de paramètres permettant de calculer la dispersion d'une substance soluble à court terme dans n'importe quelle zone du plateau continental du Nord-Ouest de l'Europe, avec une précision connue.

\section{Choix de la zone d'étude}

Plusieurs critères permettent de retenir la zone du cap de La Hague pour valider un modèle de dispersion à court terme :

- la région du cap de La Hague est soumise à une dynamique de marée particulièrement intense avec des courants pouvant dépasser $5 \mathrm{~m} / \mathrm{s}$ générant des tourbillons locaux; la topographie est également variée (nombreuses baies et caps, profondeurs variant de 10 à 100 mètres). Un modèle fonctionnant dans une zone aussi complexe pourra être considéré comme fiable ;

- les rejets de l'usine de retraitement de combustibles nucléaires usés de COGEMA-La Hague, effectués dans le Raz Blanchard au large du Cap de La Hague, sont parfaitement connus, rejet par rejet, depuis 1982 ; ils constituent la principale origine des radionucléides artificiels pour la Manche, supérieurs de deux ordres de grandeur aux autres sources pour le tritium. Ils représentent ainsi des traceurs exceptionnels pour l'étude de la dispersion en milieu marin ;

- le tritium rejeté par l'installation de La Hague, parfaitement conservatif dans l'eau de mer sous la forme HTO, est simple à échantillonner et à mesurer (mesure par scintillation liquide). Il est ainsi possible d'acquérir un grand nombre de mesures in-situ ;

- le marquage induit par les rejets de l'usine de La Hague est net : les concentrations mesurées dans le panache de l'émissaire varient de 100 à $3000 \mathrm{~Bq} / 1$ pour chaque rejet tritié, les concentrations induites le long des côtes du cap de La Hague sont d'environ 10 Bq/1, elles sont encore de $6 \mathrm{~Bq} / 1$ dans le Pas de Calais. Ces valeurs sont à comparer au «bruit de fond » des eaux entrant en Manche par l'Ouest qui varie de 0,2 à $0,3 \mathrm{~Bq} / 1$;

- le nombreuses données ont été obtenues dans le passé : marquages à l'aide de rhodamine et suivi de la dispersion durant plusieurs jours en 1962 et 1963 ; suivi de bouées dérivantes ; mesures de radiotraceurs solubles acquises par l'IPSN depuis plus de 20 ans. Elles permettent d'établir une première base de validation ;

\section{Modèle hydrodynamique}

L'outil permettant de connaître les caractéristiques des courants de marée sur l'ensemble du domaine est un modèle numérique résolvant les équations de Saint Venant, c'est à dire les équations de Navier-Stokes intégrées sur la verticale : la discrétisation du domaine de calcul est faite en différences finies suivant une grille $\mathrm{C}$ dans la classification de Arakawa. Le schéma numérique employé est dérivé de la méthode ADI (Alternate Directive Integration), il prend en compte le traitement des bancs découvrant. 
Tableau 1. Principales caractéristiques des modèles générant les conditions aux limites du modèle de La Hague en champ proche (DISPRO)

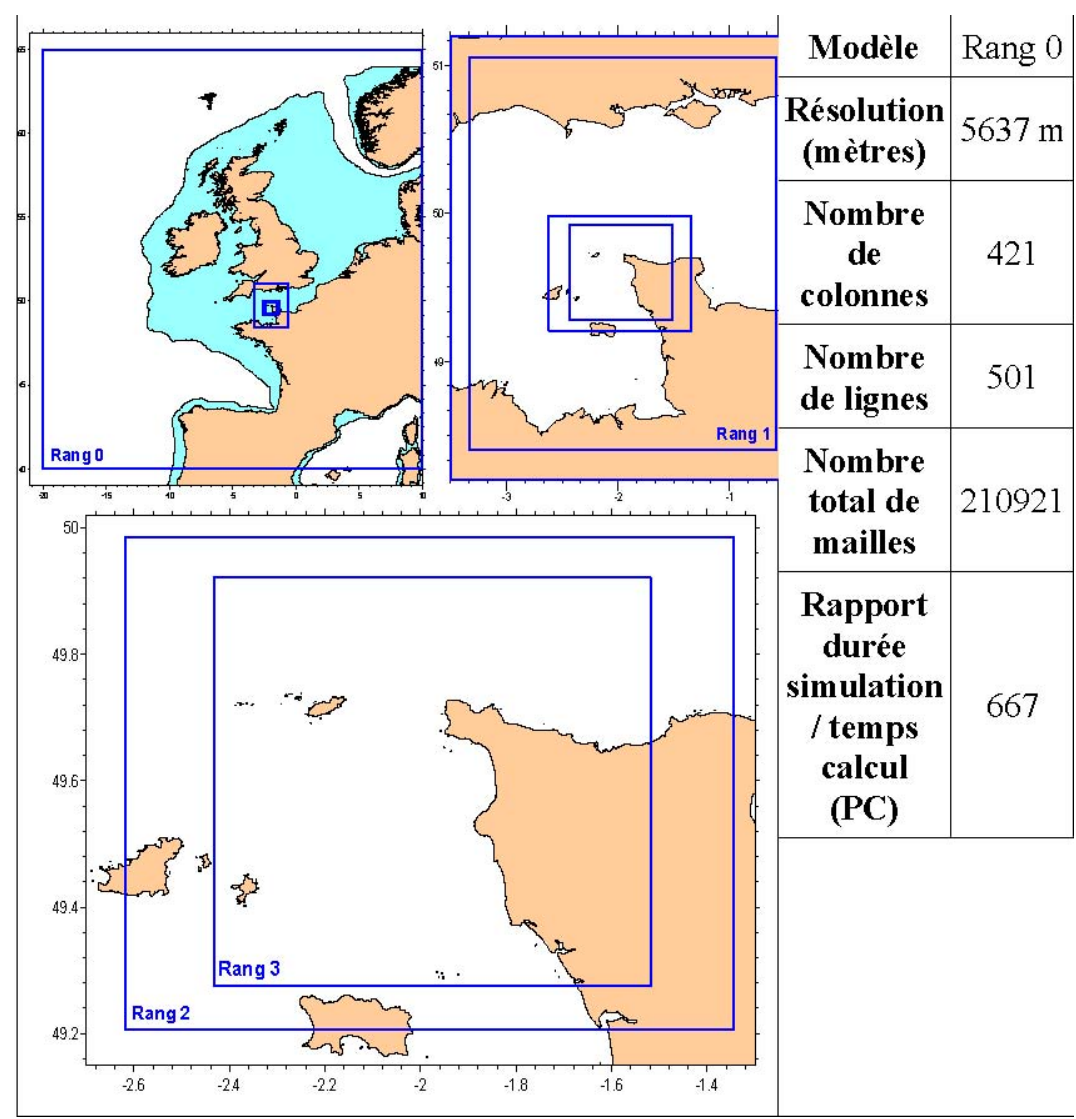

Résolution (mètres)

Nombre de colonnes

Nombre de lignes

Nombre total de mailles
Modèle $\begin{array}{ccccc}\text { Rang } & \text { Rang } & \text { Rang } & \text { Rang } \\ 0 & 1 & 2 & 3\end{array}$
$421 \quad 124 \quad 263 \quad 600$

$501 \quad 185 \quad 248 \quad 653$

$210921 \quad 22940 \quad 65224 \quad 391800$

Rapport durée simulation / temps calcul (PC)

$667 \quad 1746 \quad 134$ 
Le modèle est piloté aux limites ouvertes sur le large par la variation du niveau de la surface libre associée à la progression de l'onde de marée et sur l'ensemble du domaine par le forçage atmosphérique (vent, pression). Aucun contrôle sur les vitesses n'est effectué. Le positionnement de la surface libre est calculé par les modèles de plus grandes emprises dont les caractéristiques sont présentées dans le Tableau 1. Ils fonctionnent dans un système de coordonnées sphériques, les conditions aux limites du plus grand modèle sont établies à partir de l'atlas des composantes harmoniques de Schwiderski (1983). Les limites du modèle de grande emprise ont été repoussées au-delà de la marge continentale (à plus d'un rayon de déformation externe de Rossby) afin que le vent n'y affecte que très peu les dénivellations de la surface libre. Les conditions aux limites fermées (terre) sont de simples conditions de flux nul. Les conditions à la limite en surface correspondent aux forces de frottement exercées par le vent sur la mer. Les conditions à la limite au fond correspondent aux forces de frottement associées au déplacement de l'eau par rapport au fond.

\section{Données acquises}

\subsection{Mesures de tritium lors de campagnes en mer}

Plus de 7000 échantillons d'eau de mer ont été prélevés à bord du navire de l'INSU/CNRS « Côtes de la Manche » lors de cinq campagnes qui se sont déroulées en 2002 et 2003 . Tous ces échantillons ont été mesurés pour déterminer la concentration en tritium dissout. La stratégie d'échantillonnage a été adaptée à la chronologie réelle des rejets de l'usine COGEMA durant chaque campagne, en tenant compte des conditions réelles de dispersion.

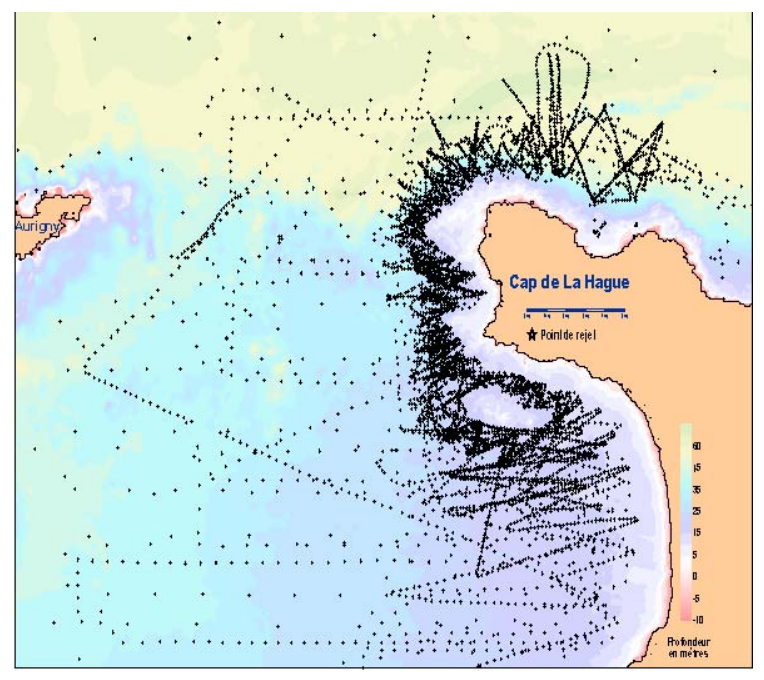

Figure 1. Localisation des prélèvements effectués lors des campagnes DISPRO

\subsection{Mesures de courants et de hauteurs d'eau}

Durant les campagnes en mer, des profils de courants ont été obtenus à l'aide d'un profileur de courants par effet doppler (ADCP) immergé dans des zones caractéristiques du point de vue hydrodynamique. Cet appareil et le sondeur du bateau ont permis d'acquérir aussi des enregistrements de la hauteur d'eau durant un ou plusieurs cycles de marée. 


\subsection{Suivis de flotteurs}

Les observations de suivis de flotteurs réalisés par la Marine Nationale en 1962 et 1963 ont été numérisées. Il s’agit de flotteurs lagrangiens drogués à 15 mètres de profondeur.

\subsection{Données météorologiques}

Les données d'analyses issues des modèles météorologiques ALADIN et ECMWF ont été collectées auprès de Météo-France. L'étalonnage du forçage du modèle associé au vent est en cours.

\subsection{Levés bathymétriques}

47000 levés bathymétriques ont été obtenus durant les campagnes DISPRO, ces levés ont fait l'objet d'une correction de la marée observée par le SHOM, afin de permettre leur intégration aux bases de données bathymétriques.

\section{Paramètres modifiés}

Le modèle donnait des résultats corrects avant d'être calibré par les données de terrain. La confrontation avec ces données a permis d'améliorer sensiblement la représentativité des simulations en modifiant certains paramètres.

\subsection{Phasage temporel du modèle}

Les mesures de courants et de hauteurs d'eau ont permis d'établir le calage temporel du modèle à 15 minutes près en se basant sur les heures de renverses de marée (Figure 2).

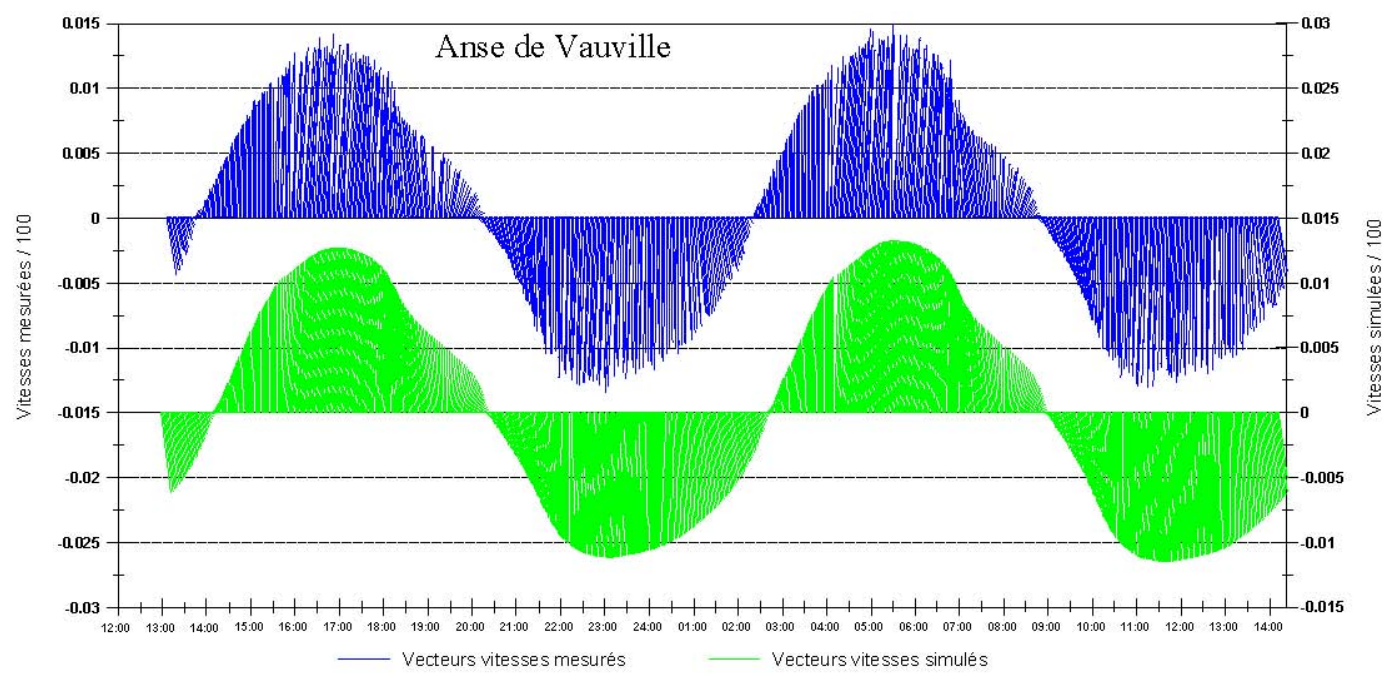

Figure 2. Courants moyens mesurés et calculés par le modèle dans l'anse de Vauville. 


\subsection{Amélioration de la bathymétrie}

Une procédure a été définie afin d'automatiser et d'améliorer ${ }_{4}$ la représentation de la bathymétrie dans les modèles hydrodynamiques, elle est décrite dans . La Figure 3 montre un détail de la bathymétrie du modèle avant et après mise à jour.
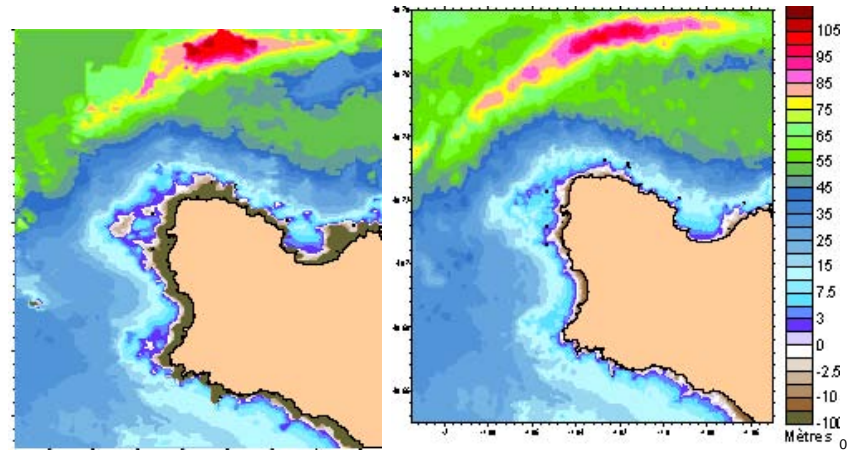

Figure 3. Bathymétrie du modèle avant et après mise à jour.

\subsection{Ajustement du coefficient de frottement}

Avec les paramètres initiaux, le modèle sous-estimait légèrement les vitesses par rapport aux observations, le coefficient de frottement de Strikler a été modifié et homogénéisé pour toutes les emprises du modèle. Après cet ajustement, l'odographe des vitesses simulées est comparable à celui obtenu avec les mesures.

\section{Résultats}

\subsection{Comparaison entre les vitesses mesurées et calculées}

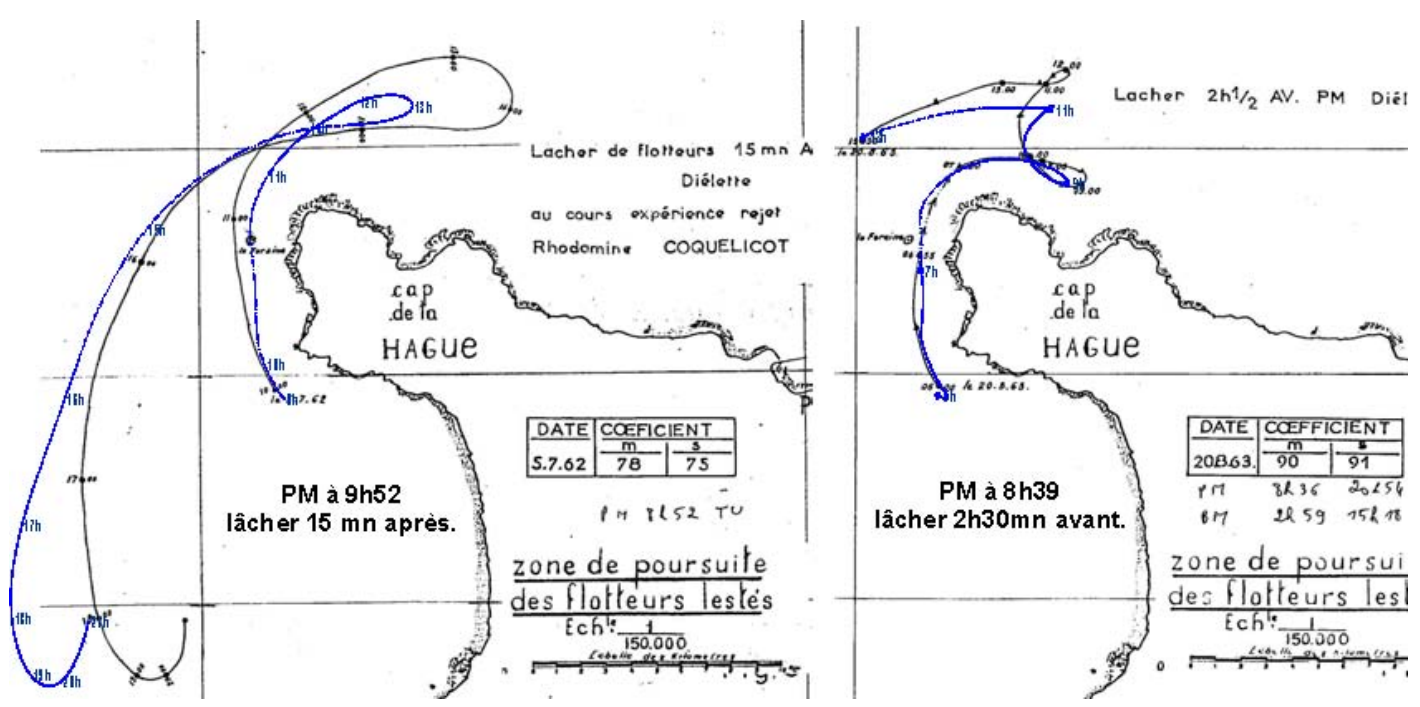

Figure 4. Trajectoires de flotteurs observées en 1962 et 1963 (traits noirs) et simulées (traits bleus). 
Les écarts entre les vitesses mesurées et simulées sont inférieurs à $10 \%$ dans les zones où l'hydrodynamique est homogène (Figure 2). Ces écarts peuvent être plus importants à proximité des côtes, en raison de la plus grande variabilité de la bathymétrie et à la moins bonne connaissance de la bathymétrie dans ces zones. Les trajectoires de flotteurs observées en 1962 et 1963 sont bien reproduites par le modèle (Figure 4). Les écarts observés au nord du cap de la Hague peuvent être logiquement attribués aux variations de la profondeur qui varie de $30 \mathrm{~m}$ à 90 $\mathrm{m}$ : les trajectoires des flotteurs drogués à 15 mètres ne peuvent pas représenter le flux d'eau moyen calculé par le modèle.

\subsection{Comparaison entre les concentrations en tritium mesurées et calculées}

Durant les cinq campagnes DISPRO, neuf rejets ont pu être suivis dans des conditions hydrodynamiques très variables (vent faible à fort, courant portant vers le sud ou vers le nord au moment du rejet). Entre 500 et 1000 mesures de tritium dissout ont été obtenues pour chaque rejet. Le modèle simule la dispersion avec une bonne précision durant les six heures qui suivent le début du rejet. Les résultats sont moins précis lorsque le panache est entraîné vers le sud, ce qui peut s'expliquer par une moins bonne connaissance de la bathymétrie sur le plateau rocheux situé au sud de l'émissaire (plateau des Huquets). La Figure 5 illustre la capacité du modèle à reproduire les panaches mesurés

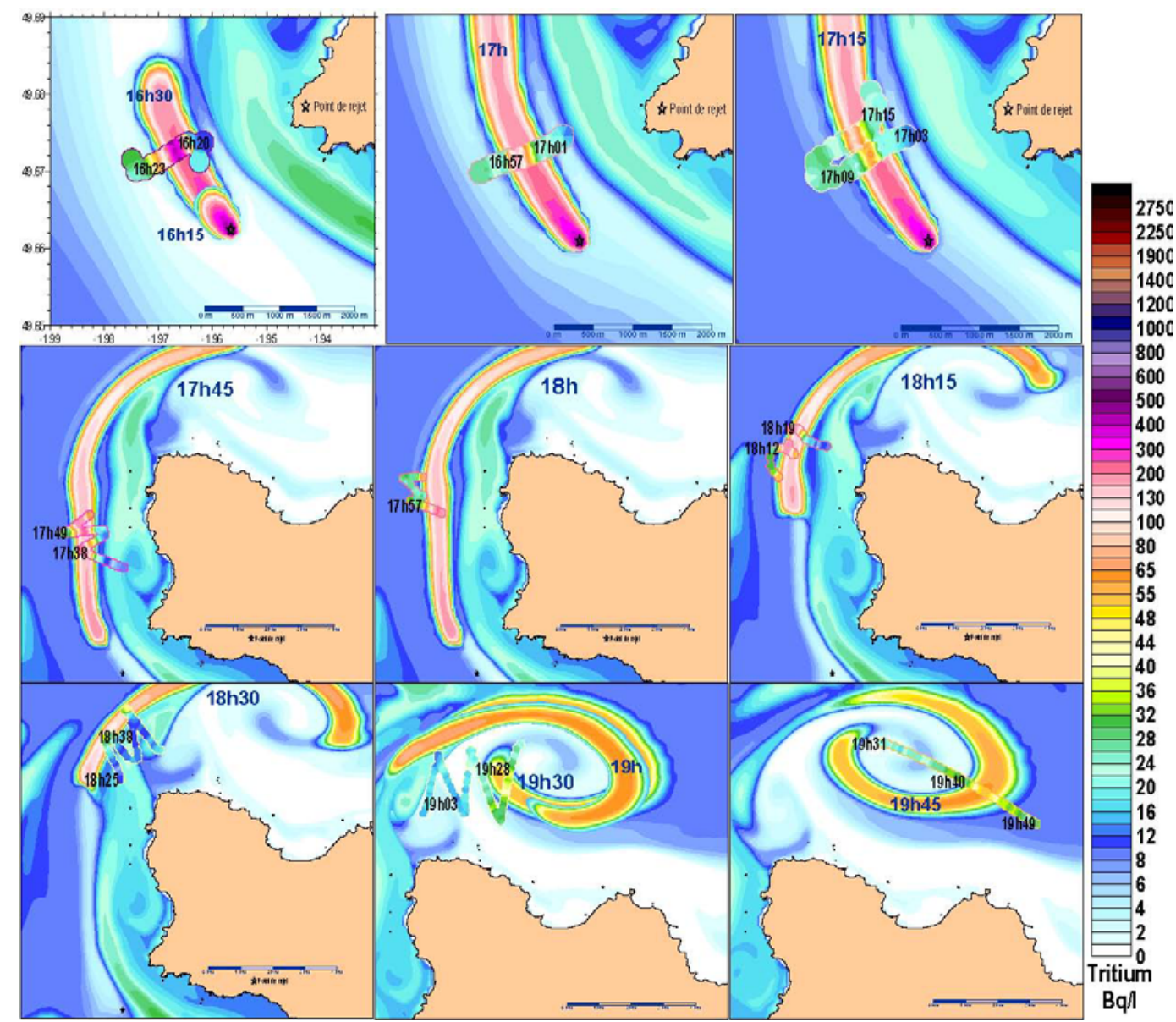

Figure 5. Comparaison entre les concentrations simulées (arrière plan, heures en bleu) et mesurées (séries de points colorés, heures en noir) le 12 juin 2003 entre $16 \mathrm{~h}$ et $20 \mathrm{~h}$. 


\section{Conclusion}

Les données acquises constituent une base unique pour affiner les paramètres de dispersion de modèles pour des conditions réelles de rejet, de marée et de vent, dans une zone où la dynamique des courants est particulièrement active (le raz Blanchard au large du Cap de La Hague).

Dans l'état actuel du projet, le modèle peut être considéré comme fiable pour représenter la dispersion durant les six heures suivant un rejet. Au-delà l'influence du vent devient significative. Les travaux en cours visent à préciser cette influence de manière à obtenir une précision comparable durant les jours et les semaines suivant un rejet. Pour des simulations de la dispersion plus longues, d'autres outils permettent de représenter la dispersion durant plusieurs années à l'échelle de la Manche et de la Mer du Nord .

Les résultats acquis lors des campagnes DISPRO doivent être généralisés à l'ensemble de la Manche au moyen de modèles de résolution plus grossière. Les paramètres de dispersion établis avec un modèle de 100 mètres de résolution seront testés et ajustés avec des modèles de moins grande précision $(0,3 ; 1,6$ et $5 \mathrm{~km}$ de résolution). L'objectif ultime est de disposer de modèles hydrodynamiques ayant des incertitudes connues pour la simulation de la dispersion de substances solubles naturelles ou d'origine anthropique avec des temps de calcul raisonnables. Ces modèles sont destinés notamment à reproduire la dispersion de rejets industriels chroniques ou résultant d'accidents

\section{Remerciements}

Nous tenons à remercier particulièrement les équipages du navire de l'INSU/CNRS « Côtes de la Manche », qui ont permis de réaliser les prélèvements dans des conditions difficiles, ainsi que l'équipe de l'UMR 6143 Morphodynamique Continentale et Côtière de l'Université de Rouen, pour les mesures courantologiques.

\section{Références}

1.Breton M. and Salomon J.C., (1995). A 2D long-term advection-dispersion model for the Channel and southern North Sea. Part A: validation through comparison with artificial radionuclides. Journal of Marine Systems, 6 (5-6), 495-514.

2.Bailly du Bois P. and Guéguéniat P., (1999). Quantitative assessment of dissolved radiotracers in the English Channel: sources, average impact of la Hague reprocessing plant and conservative behaviour (1983, 1986, 1988 and 1994). Continental Shelf Research ; FluxManche II Dedicated, 19, 1977-2002.

3. Bailly du Bois P., (2003). TRANSMER, modèle de simulation des transferts de radionucléides en milieu marin : choix des options, architecture, validation et fonctionnalités. Rapport IRSN/DPRE/SERNAT/ 2003-04 188 p.

4. Bailly du Bois P. (2004). Construction du fichier bathymétrique du modèle hydrodynamique DISPRO (dispersion d'effluents dans le champ proche d'un émissaire de rejet en mer). Rapport IRSN/DEI/SECRE/LRC 2004-01 17 p. 